\title{
Effect of shell thickness deviation on buckling load of free-form shells under uncertainties
}

\author{
Bingbing San, Liwei Yin \\ College of Civil and Transportation Engineering, Hohai University, Nanjing, China \\ sanbingbing@163.com
}

Keywords: free-form shells, shell thickness deviation, buckling load, uncertainties

Abstract. Uncertain errors induced in construction process could affect the stability of free-form shells. Considering shell thickness deviation, the stochastic characteristics of buckling loads are investigated using Latin hypercube sampling coupled with Newton-Raphson method. In addition, a sensitivity analysis is conducted to quantize the effect of the thickness deviation on buckling loads of free-form shells.

\section{Introduction}

In recent years, free-form shells have been widely adopted in civil engineering. 'Free-form' means their geometric shapes cannot be described by any analytical functions. Compared to traditional shells, free-form shells provide more options to architects, and thus become a new trend of spatial structures. The stability issue is a key problem in the designing of shells. The studies on traditional shells have shown that uncertain factors, such as thickness deviation, material deviations, etc., have significant influence on stability. Compared with traditional shells, the geometric shapes of the free-form shells are more complicated, which will lead to more stochastic errors in the process of construction. However, the researches on stability of free-form shells are still limited, and most of them are based on deterministic analysis, without considering the influence of uncertainties.

Focusing on the shell thickness deviation, this paper carries out a stochastic analysis and a sensitivity analysis to investigate the effect of thickness deviation on critical buckling load of free-form shells. Shapes of shells are obtained by an optimization method. Latin Hypercube Sampling (LHS) method is adopted for random sampling. The critical buckling loads of shell is calculated by Newton-Raphson method.

\section{Analysis models of free-form shells}

Shape optimization of free-form shells. The Non-Uniform Rational B-Spline (NURBS) method is used to describe the geometric shape of the free-form shells. The shapes are optimized by adjusting the coordinates of the control points using the gradient method. In this paper, the optimization objective is set up as minimum strain energy. The optimal shells will be adopted in the stochastic and sensitivity analysis.

Two free-form models are analyzed here, which are named Mode 1 and Mode 2, respectively. Both of them are continuous shells. The material is assumed to be concrete. Elastic modulus is 30GPa. Poisson's ratio is 0.167 . The design thickness is $0.14 \mathrm{~m}$.



(a) control points

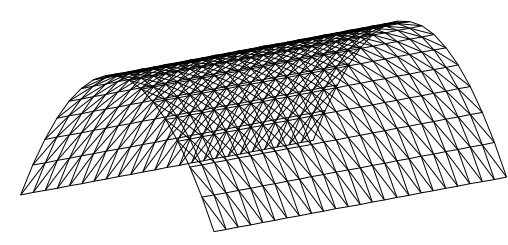

(b) initial shape

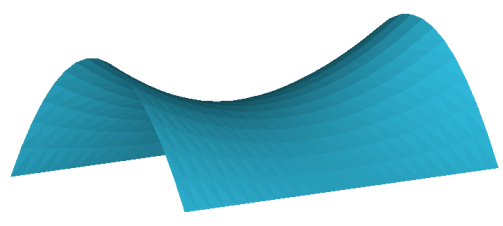

(c) optimal shape

Figure 1: Control points, initial shape and optimal shape of Model 1 
The control points and initial shape of Mode 1 is shown in Fig. 1 (a, b). The length and width is $12 \mathrm{~m}$ and $8 \mathrm{~m}$, respectively. The initial shape is determined as a parabolic columnar surface, with the maximum height of $4 \mathrm{~m}$. The shell is assumed to be under vertical uniform load of $3.5 \mathrm{kN} / \mathrm{m} 2$. The boundaries along length are simply supported while the other boundaries are free. The gradient method is adopted to solve for the optimal shape. The result is shown in Fig. 1 (c).

The control points and initial shape of Mode 2 is shown in Fig. 2 (a). The length and width is $20 \mathrm{~m}$ and $16 \mathrm{~m}$, respectively. All boudaries are simply supported. The optimization is carried out and the optimal shape is obtained (Fig. 2 (b)). It can be seen that, since the number of control points is larger than that of Model 2, the shape of this shell is more various.



(a) control points and initial shape

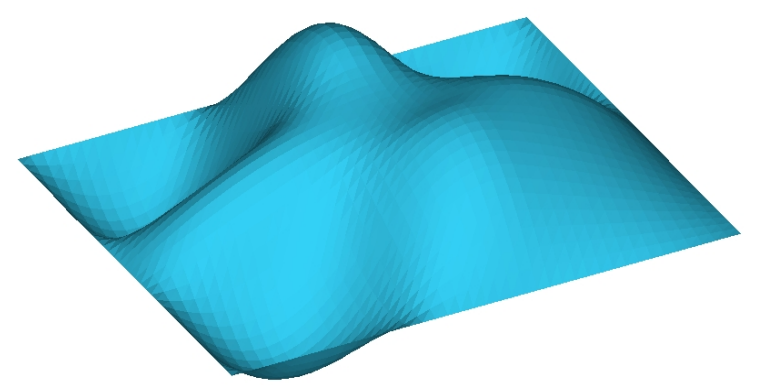

(b) optimal shape

Figure 2: Control points, initial shape and optimal shape of Model 2

Stochastic model. Nowak and Carr [1] have analyzed the statistics of shell thickness deviation, which shows that shell thickness deviation follows normal distribution. According to Code for acceptance of constructional quality of concrete structures (GB50204-2015) [2], the allowable construction error of concrete structural members is $-5 \mathrm{~mm} \sim+8 \mathrm{~mm}$. So, the range of shell thickness deviation, which is defined as the ratio of thickness error to design thickness, is determined as $-3.571 \% \sim+5.714 \%$, with a guarantee rate of $95 \%$. Consequently, the mean value $\mu$ of shell thickness deviation is $+1.071 \%$ and the standard deviation $\sigma$ is 0.02369 .

\section{Stochastic analysis and sensitivity analysis method}

The critical buckling load analyses are coupled with Latin hypercube sampling (LHS) [3] to investigate the stochastic characteristics of deviation effects. The procedure of LHS can be divided into two main steps, namely, sampling and permutation. The objective of sampling is to produce representative samples to describe the distribution of each input random variable. Permutation aims at reducing the correlations between samples of different input random variables. The sample size $n$ is determined as 400 here.

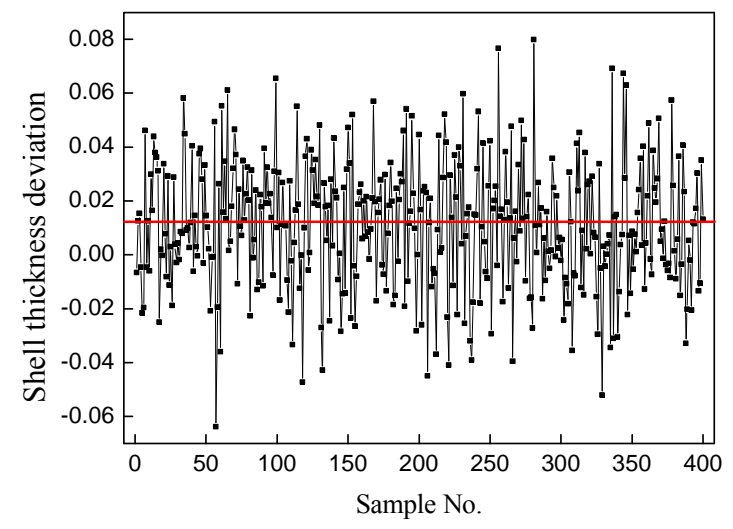

Figure 3: Sampling results of shell thickness deviation The sampling results are shown in Fig. 3.

Newton-Raphson method is adopted to solve the critical loads of samples. Furthermore, the non-dimensional sensitivity is calculated to quantize the effect of the thickness deviations on buckling loads. The sensitivity function is defined as follows:

$$
S(a)=\left|\frac{\Delta P}{P}\right| /\left|\frac{\Delta a}{a}\right|=\left|\frac{\Delta P}{\Delta a} \times \frac{a}{P}\right|
$$


where, $a$ is shell thickness deviation and $P$ is buckling load of shells.

\section{Results and discussion}

Taking the random variation of shell thickness deviation into account, the stochastic and sensitivity analysis is performed for Model 1. The critical buckling loads of 400 samples are calculated by Newton-Raphson method and given in Fig. 4. The mean value $\mu$ of critical buckling load is solved as $3511.84 \mathrm{kN} / \mathrm{m}^{2}$, and the standard deviation $\sigma$ is solved as $207.58 \mathrm{kN} / \mathrm{m}^{2}$. The coefficient of variation $(\sigma / \mu)$ is $5.911 \%$.

The cumulative probability distribution is described in Fig. 5. Within its variation range, its distribution function is fitted as Eq. 2, which is approximately a Gaussian curve.

$$
f(x)=0.9508 e^{-\left(\frac{x-4132}{476.7}\right)^{2}}+0.4997 e^{-\left(\frac{x-3613}{322.2}\right)^{2}}
$$

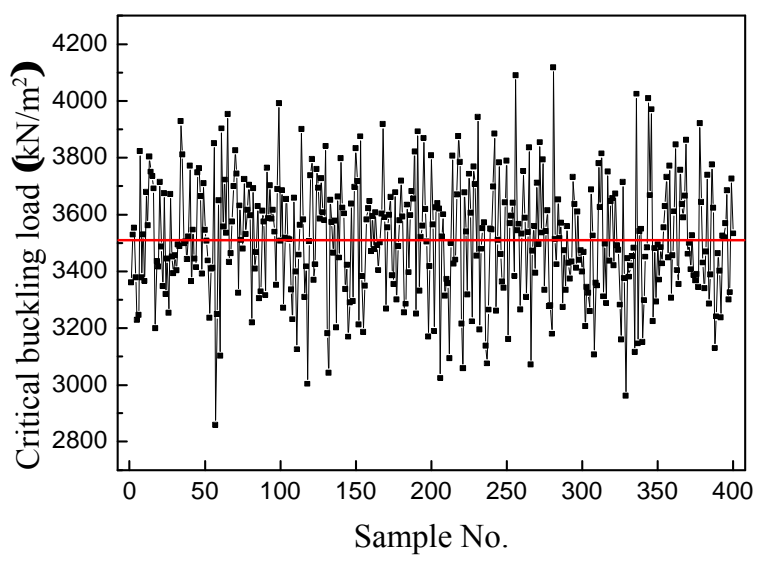

Figure 4: Critical buckling load of Model 1

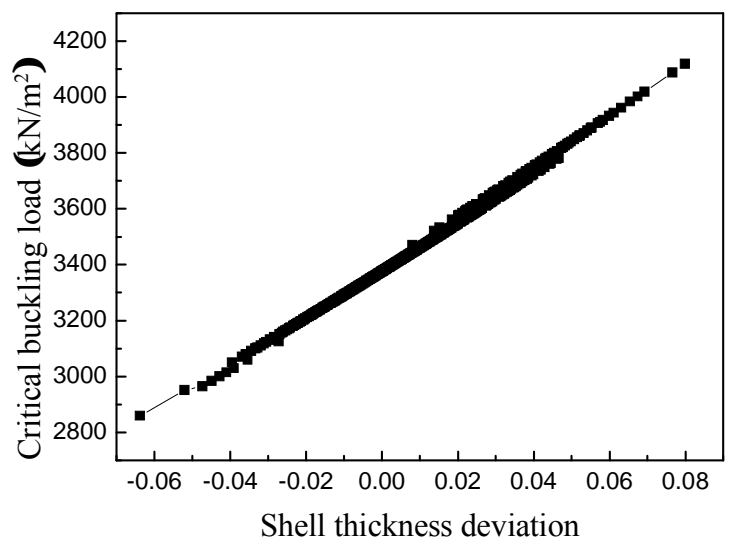

Figure 6: Relationship between critical buckling load and shell thickness deviation (Model 1)

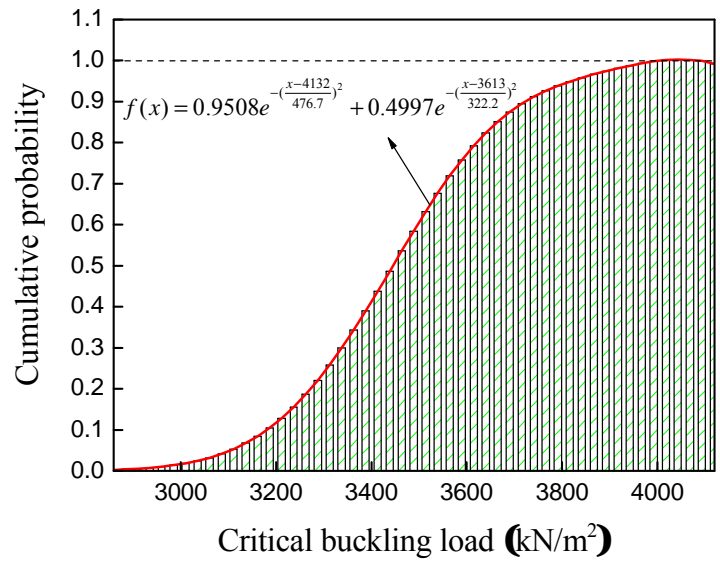

Figure 5: Cumulative probability distribution of critical buckling load of Model 1

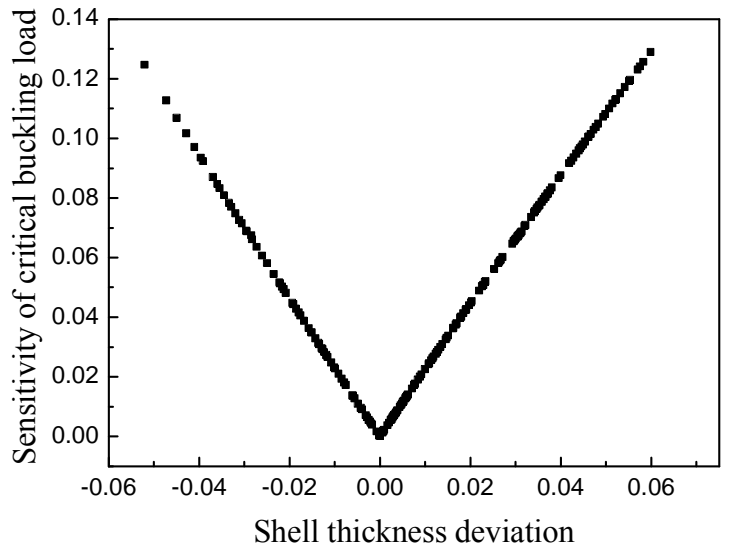

Figure 7: Sensitivity of critical buckling load to shell thickness deviation(Model 1)

Fig. 6 shows the relationship between critical buckling load and shell thickness deviation of Model 1 . It can be seen that the critical loads are increased when the deviation is varied from -0.06 to 0.08 . The sensitivity values are shown in Fig. 7. It varies within $0.0 \sim 0.14$ and mean value is 0.04852 . The similar analysis is conducted on Model 2. The critical buckling loads of 400 samples of Model 2 are shown in Fig. 8. The mean value $\mu$ and standard deviation $\sigma$ is solved as $2450.56 \mathrm{kN} / \mathrm{m}^{2}$ and $98.88 \mathrm{kN} / \mathrm{m}^{2}$, respectively. The coefficient of variation is $4.035 \%$. 
Also, the cumulative probability distribution of critical buckling load of Model 2 is described in Fig. 9. Within its variation range, its distribution function is fitted as Eq. 3, which is approximately a Gaussian curve.

$$
f(x)=0.9199 e^{-\left(\frac{x-2762}{220.7}\right)^{2}}+0.5214 e^{-\left(\frac{x-2523}{157.8}\right)^{2}}
$$



Figure 8: Critical buckling load of Model 2



Figure 9: Cumulative probability distribution of critical buckling load of Model 2

The relationship between critical buckling load and thickness deviation of Model 2 is also obtained, which shows the same tendency to Model 2 . The sensitivity values of Model 2 is $0.0 \sim 0.15$ with a mean value of 0.04568 , which are closer to that of Model 2.

\section{Conclusions}

In this paper, a stochastic and sensitivity analysis is conducted to investigate the effect of thickness deviation on critical buckling load of free-form shells. Two free-form models are involved. The stochastic characteristics are obtained. It shows that the cumulative probability distribution of critical buckling load of free-form shells is approximately a Gaussian curve with respect to uncertain shell thickness deviations. And the coefficient of variation is around 5.0\%, which is in the range of low variation.

\section{Acknowledgements}

This work is sponsored by the National Natural Science Foundation of China (Grant no. 51578211). The authors are grateful for this financial support.

\section{References}

[1] Nowak A.S. and Carr R.I, Sensitivity Analysis for Structural Errors. Journal of Structural Engineering, 1985, 111(8):1734-1746.

[2] GB 50204-2015: Code for acceptance of constructional quality of concrete structures, China Architecture \& Building Press. 2015.

[3] San B.B., Yang Q.S. and Yin L.W., Stochastic and sensitivity analysis of shape error of inflatable antenna reflectors, Acta Astronautica, 2017,132,170-176. 